\title{
Polarization switching in vertical-cavity surface emitting lasers observed at constant active region temperature
}

\author{
J. Martín-Regalado, ${ }^{a}$ J. L. A. Chilla, and J. J. Rocca \\ Departament of Electrical Engineering and Center for Optoelectronic Computing Systems, \\ Colorado State University, Fort Collins, Colorado 80523 \\ P. Brusenbach \\ Vixel Corporation, Broomfield, Colorado 80021
}

(Received 28 February 1997; accepted for publication 16 April 1997)

\begin{abstract}
Polarization switching in gain-guided, vertical-cavity, surface-emitting lasers was studied as a function of the active region temperature. We show that polarization switching occurs even when the active region temperature is kept constant during fast pulse low duty cycle operation. This temperature independent polarization switching phenomenon is explained in terms of a recently developed model. (C) 1997 American Institute of Physics. [S0003-6951(97)00725-0]
\end{abstract}

The optical and electrical properties of vertical cavity surface emitting lasers (VCSELs): single-longitudinal mode emission, low-threshold current, low-divergence circular output beam, make these devices attractive for applications such as optical-fiber and free-space communications, optical recording, etc. Although VCSELs often show stable linear polarization emission with the vector field preferentially oriented along one of two orthogonal directions associated with the crystal axes, ${ }^{1}$ a drawback of many VCSELs is a polarization instability, known as polarization switching (PS), which occurs as the injection current is increased close beyond the lasing threshold. ${ }^{2} \mathrm{~A}$ better understanding of the mechanisms which influence the polarization dynamics in VCSELs may lead to improved performance for polarization-sensitive applications such as optical switching.

An explanation for polarization switching in VCSELs has been offered by Choquette $e t a l{ }^{3}$ based on the fact that self heating of the devices by the injected current changes the relative spectral alignment between the laser gain and the cavity resonances of nondegenerate polarization states. An additional mechanism for polarization state selection arises from the difference in the population of the magnetic sublevels of the conduction and heavy-hole valence bands in a quantum-well VCSELs. ${ }^{4}$ This population difference is annihilated by spin-flip relaxation processes in a time scale of tens of picoseconds, ${ }^{5}$ which is comparable to the photon lifetime and thus slow enough to have an important effect on the dynamics. Within the framework of a theory including magnetic sublevels, ${ }^{6,7} \mathrm{PS}$ is expected as the injection current is scanned above the lasing threshold, even when the gain difference between the linearly polarized modes (active region temperature) is kept constant.

In this letter we report a systematic study of the temperature dependent polarization switching behavior which can be observed during single mode emission in gain-guided vertical-cavity surface emitting lasers. To discriminate between the effects produced by changes in the pump current (and the associated changes in the nonlinear coupling of the field and the carrier number through gain and dispersion) and those produced by changes in the current-induced self heat-

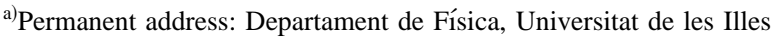
Balears, E-07071 Palma de Mallorca, Spain. ing we have taken advantage of the relatively slow time scale of thermal effects. ${ }^{8}$ We first performed continuous wave (cw) experiments using a current ramp duration of a few seconds that gives the temperature time to stabilize for each value of the pump current and dissipated power. We also performed experiments in which the current ramp used was short as compared to the thermal response time so that the temperature of the active region stays constant during the current scan. In this latter case the observed polarization switching cannot be attributed to thermal effects. The nonthermal polarization switching is consistent with predictions from the model incorporating magnetic sublevels. ${ }^{6,7}$

The lasers used in this study are proton-implanted AlGaAs/GaAs multiple quantum well VCSELs with cavity mirrors consisting of AlAs/AlGaAs distributed Bragg reflectors, which had 19 periods for the $p$-doped top mirror and 29 periods for the $n$-doped bottom mirror. ${ }^{9}$ Measurements were performed on a linear array of cylindrically symmetric devices with active region diameter of $15 \mu \mathrm{m}$ and top contact window diameter of $12 \mu \mathrm{m}$, all of them operating around $850 \mathrm{~nm}$. The array was mounted on a thermoelectric cooler to control the substrate temperature. The $\mathrm{cw}$ measurements were performed using a HP-4145A semiconductor parameter analyzer (SPA) to simultaneously monitor the injected current, the voltage across the junction, and the optical power in both polarizations. The latter were measured by using two broad area photodetectors located after a polarizer beam splitter. The thermal properties of the VCSELs were studied by monitoring the dissipated power dependence of the emission wavelength at different substrate temperatures. ${ }^{10}$ The active region temperature was obtained from the measured dissipated power and the measured value of $1.52{ }^{\circ} \mathrm{C} / \mathrm{mW}$ for the thermal resistance.

From the set of 8 VCSELs analyzed, three of them (VCSELS A, B, C) showed PS within the range of temperatures measured $\left(-30\right.$ to $\left.50{ }^{\circ} \mathrm{C}\right)$, while the rest of the devices had stable linear polarization for fundamental mode emission. The frequency splitting (birefringence) between the linearly polarized modes, measured with a high resolution monochromator in $\mathrm{cw}$ operation for currents closely below threshold, was in the range $\Delta \nu=18-20 \mathrm{GHz}$ for devices showing PS, while for the rest was unresolved (except for one). In all the switchings we observed that the wavelength 


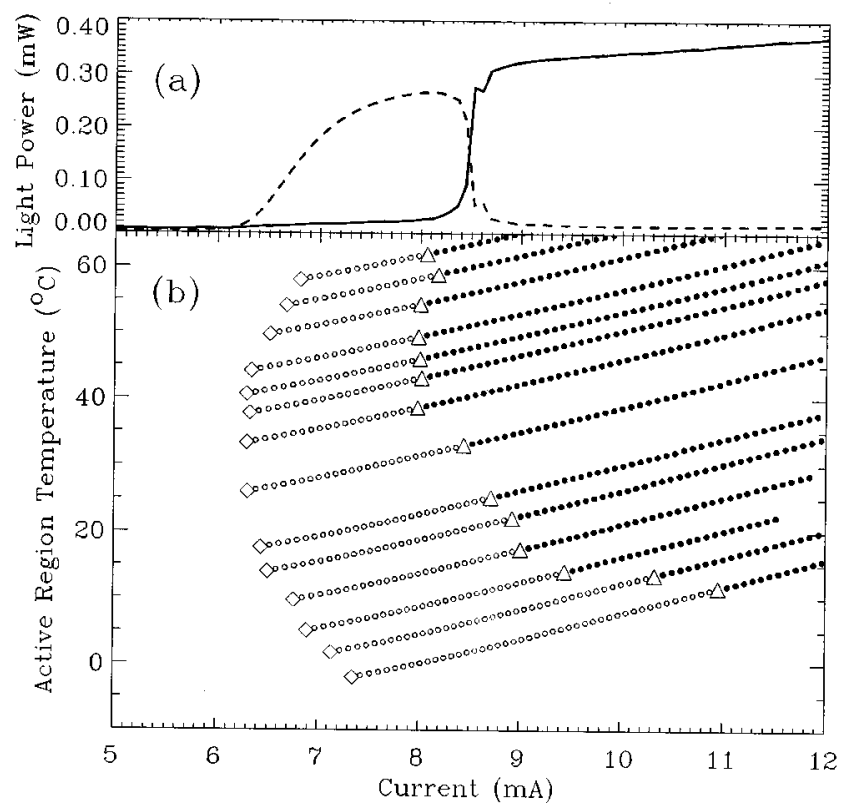

FIG. 1. (a) Polarized light-current characteristics at $8{ }^{\circ} \mathrm{C}$ substrate temperature under cw conditions. (b) Active region temperature vs. injected current diagram for $\mathrm{cw}$ operation. Threshold currents are indicated by rhombs, and switching currents by triangles. The open (solid) circles corresponds to linear short (long) wavelength polarization emission.

of the linearly polarized mode selected at threshold was lower than that of the dominant mode after the switching.

In the following, we will concentrate on the VCSEL showing PS in the whole range of temperatures analyzed (VCSEL A), the other two VCSELs (B and C) had a similar behavior but in narrower temperature ranges. Figure 1(a) shows the light vs. current (LI) for VCSEL A taken at $8{ }^{\circ} \mathrm{C}$ substrate temperature. PS is abrupt and occurs at $8.5 \mathrm{~mA}$. In order to characterize the PS behavior under cw operation we have plotted in Fig. 1(b) the domains of polarization dominance taking into account the temperature rise of the active region which occurs as the current is increased. The threshold current shows the characteristic dependence with the active region temperature unique to VCSELs, ${ }^{3}$ which is due to the relative alignment of the cavity resonances with the gain spectrum in such a way that the minimum threshold current occurs at the temperature where the cavity resonances are aligned with the gain peak. The map in Fig. 1(b) shows the general features already reported in Ref. 3, namely the polarization switches from the mode with shorter wavelength for low currents to the mode with longer wavelength for larger currents. The current at which PS occurs increases as the temperature is decreased. Notice, however that the boundary between the dominance regions of the different polarizations is not a horizontal line corresponding to the temperature at which the cavity resonances exactly match the material gain, indicating the existence of additional effects that complement the relative spectral position of the cavity resonances and material gain in determining the polarization characteristics of VCSELs.

The effects of the temperature on the polarization dynamics of VCSELs were minimized by performing light and voltage vs. current (LIV) measurements using short current ramps at low duty cycle. After determining the thermal re-

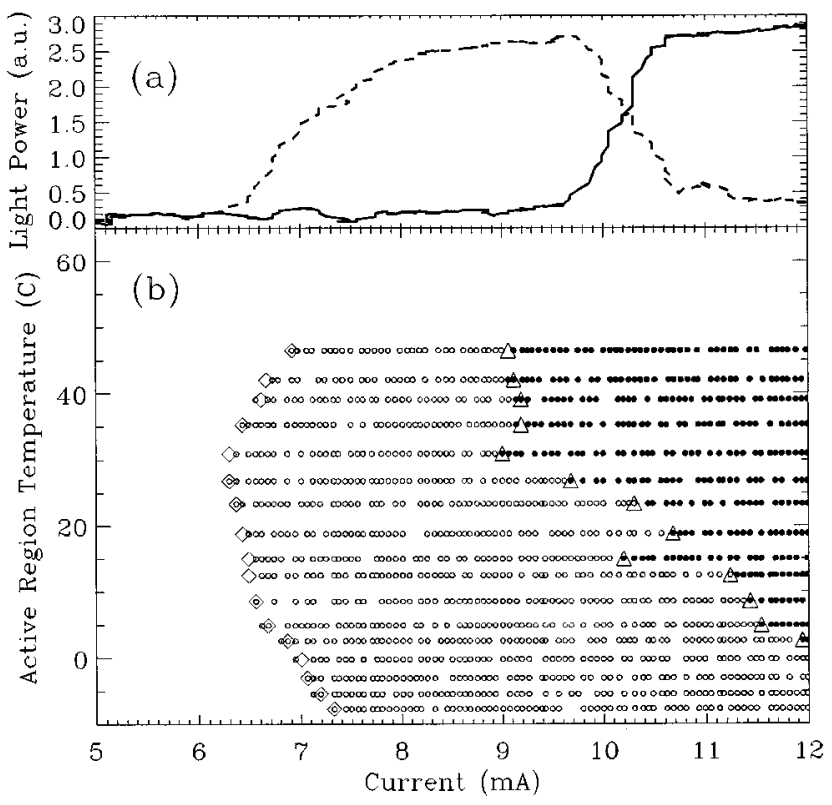

FIG. 2. Same as Fig. 1 for fast current ramp operation with $3 \mathrm{~mA}$ prebias current.

sponse time to be about $1 \mu \mathrm{s}$, the duration of the ramps was chosen to be $100 \mathrm{~ns}$. As the typical times in carrier-field interaction (relaxation oscillations) are around $1 \mathrm{~ns}$ this ramp duration is slow enough to ensure that the measurements are taken in a quasisteady situation while being fast enough to avoid the effect of self-heating. The current source used consisted of a dc prebias and a fast $(100 \mathrm{~ns}, 20 \mathrm{kHz})$ current ramp generator connected to the VCSEL through a bias-tee network. The injected current and the voltage across the VCSEL were measured using a $50 \Omega$ load resistance while the light power was measured independently for both polarizations using a fast $(\sim 1 \mathrm{~ns})$ photodiode. All the signals were monitored using a digital oscilloscope $(100 \mathrm{Ms}, 100 \mathrm{MHz}$ bandwidth) and taking into account the different delays in the transmission lines. The fact that the measurements were taken at constant active region temperature was verified by time-resolved spectrum measurements of the VCSEL output which showed a small blue shift $(<0.5 \AA)$ of the wavelength emission during the pulse duration which can be attributed to the index reduction as the carrier density increases with the injected current.

A typical LI measurement obtained for VCSEL A during fast ramp excitation at $8{ }^{\circ} \mathrm{C}$ substrate temperature using a prebias current of $3 \mathrm{~mA}$ is shown in Fig. 2(a). The data shows polarization switching, providing the first clear experimental evidence for the effect predicted by the theory in Refs. 6 and 7, which is similar to that in Fig. 1(a) but here occurs around $10 \mathrm{~mA}$. To characterize the temperature dependence of the PS behavior under fast current excitation we plot in Fig. 2(b) the domains of polarization dominance at different active region temperatures. In this figure the active region temperature, given by the substrate temperature and the self-heating produced by the prebias current, does not change during the current ramp. The dependence of the threshold current follows that of Fig. 1(b), as can be expected since the threshold current depends mainly on the mismatch of the cavity resonances and the gain peak. On the 
other hand, the regions of dominance of each polarization in Fig. 2(b) are similar to those obtained in the cw experiments, although not the same, providing further evidence of the action of a different switching mechanism. Below we provide a possible explanation for the observed nonthermal PS, based on a recently proposed theoretical model ${ }^{6,7}$ that shows good agreement with the measurements.

A nonthermal mechanism for polarization state selection arises from the difference in the population of the magnetic sublevels of the conduction and heavy-hole valence bands in quantum-well VCSELs. As the natural emission channels are circularly polarized, the amplitude-phase coupling associated with the linewidth enhancement factor results in energy coupling between the linearly polarized modes for non-zero values of the spin-sublevel population difference. Fluctuations in population difference and the amplitude of the weak mode relax to equilibrium (zero) through damped oscillations whose frequency is affected by the injected current. When this oscillatory decay comes in resonance with the birefringent splitting of the mode frequencies, the phase relations become favorable for energy transfer between the polarization modes and the damped oscillations become undamped. This makes the dominant mode unstable and the polarization switches through a phenomenon known as phase instability at a Hopf Bifurcation. The theoretical model of this mechanism for PS, described in detail in Ref. 7 is based on a rate equation model ${ }^{4}$ which takes into account the vector nature of the laser field, saturable dispersion, VCSEL anisotropies (birefringence and dichroism), a carrier dynamics associated with the different magnetic sublevels of the conduction and heavy hole valence bands in quantum-well VCSELs. The model predicts a linear dependence of the switching current normalized to threshold with the gain difference between the linearly polarized modes $\left(\gamma_{a}\right)$ given by:

$$
\mu_{s w} / \mu_{t h}=1+\frac{2\left(\gamma_{s}^{2}+4 \gamma_{p}^{2}\right) \gamma_{a}}{\kappa\left(2 \alpha \gamma_{p}-\gamma_{s}\right) \gamma},
$$

where $\kappa$ is the field decay rate, $\alpha$ is the linewidth enhancement factor, $\gamma_{p}=\pi \Delta \nu$ is the VCSEL birefringence, and $\gamma_{s}$ is the spin-flip decay rate. In order to associate the dependence of the gain difference on the active region temperature, we have assumed a parabolic dependence of the gain spectrum around the gain peak. Then, it is straightforward to demonstrate that the gain difference between two nondegenerate modes with the same transverse pattern changes linearly with the temperature, taking into account the linear dependence of the emission wavelength on the active layer temperature. The solid line in Fig. 3 represents the fitting of the experimental data using typical parameters: $\kappa=300 \mathrm{~ns}^{-1}, \alpha=3, \gamma^{-1}=1 \mathrm{~ns}$, $\gamma_{p}=60 \mathrm{~ns}^{-1}$, and $\gamma_{s}=100 \mathrm{~ns}^{-1}{ }^{11}$ As can be observed in Figs. 1 and 2, the short wavelength polarization mode still dominates at threshold at temperatures where the material gain favors the orthogonal mode due to the existence of an

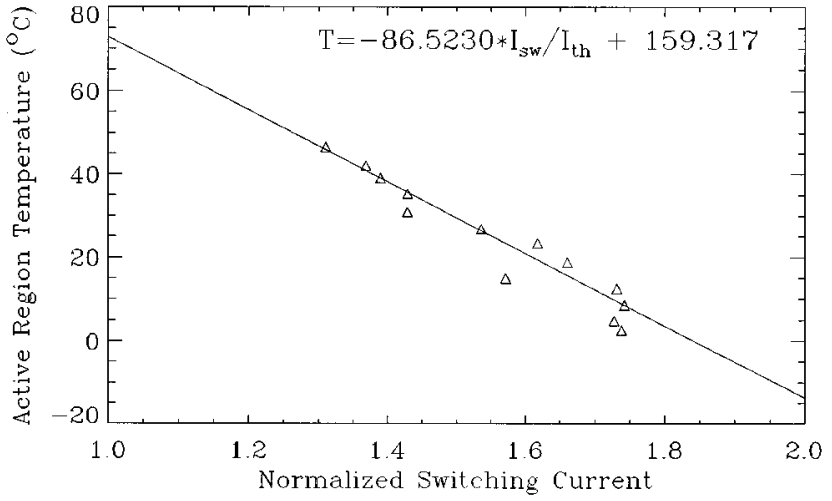

FIG. 3. Dependence of the switching current normalized to the threshold current vs the active region temperature. The triangles are the measured data. The solid line is the calculated result.

intrinsic modal gain/loss anisotropy favoring the shorter wavelength mode. We have estimated from the data of Fig. 3 the value of intrinsic gain anisotropy to be $\gamma_{a}=0.1 \mathrm{~cm}^{-1}$, in good agreement with Ref. 12.

In summary, PS at constant active region temperature has been observed. This result indicates that new physical mechanisms, unrelated to temperature changes, also cause PS in VCSELs. The dependence of the switching current on the active layer temperature shows a good agreement with a recently developed model which predicts similar switching as a consequence of the combined effect of the $\alpha$-parameter, VCSEL anisotropies, and spin-flip mixing processes.

The authors thank N. B. Abraham for helpful discussions and comments. We acknowledge financial support from CICYT (Spain) Project No. TIC95/0563 and from the Center for Optoelectronic Computer Systems through N.S.F. Grant No. EEC-9015128 and Colorado Advanced Technology Institute Grant No. GEA95-0002.

${ }^{1}$ K. D. Choquette and R. E. Leibenguth, IEEE Photonics Technol. Lett. 6 , 40 (1994).

${ }^{2}$ S. Jiang, Z. Pan, M. Dagenais, R. A. Morgan, and K. Kojima, Appl. Phys. Lett. 63, 3545 (1993).

${ }^{3}$ K. D. Choquette, R. S. Jr., K. Lear, and R. E. Leibenguth, IEEE J. Sel. Top. Quantum Electron. 1, 661 (1995).

${ }^{4}$ M. S. Miquel, Q. Feng, and J. Moloney, Phys. Rev. A 52, 1728 (1995).

${ }^{5}$ T. C. Damen, et al., Phys. Rev. Lett. 67, 3432 (1991).

${ }^{6}$ J. Martín-Regalado, M. S. Miguel, N. B. Abraham, and F. Prati, Opt. Lett. 21, 351 (1996).

${ }^{7}$ J. Martín-Regalado, M. S. Miguel, N. B. Abraham, and F. Prati, IEEE J. Quantum Electron. 33, 765 (1997).

${ }^{8}$ G. Hasnain, K. Tai, L. Yang, Y. H. Wang, R. J. Fisher, J. D. Wynn, B. Weir, N. K. Dutta, and A. Y. Cho, IEEE J. Quantum Electron. 27, 1377 (1991).

${ }^{9}$ Vixel Corporation, 325 Interlocken Parkway, Broomfield, CO 80021.

${ }^{10}$ D. B. Young, J. W. Scott, F. H. Peters, M. G. Peters, M. L. Majewski, B. F. Thibeault, S. W. Corzine, and L. A. Coldren, IEEE J. Quantum Electron. 29, 2013 (1993).

${ }^{11}$ A. K. J. van Doorn, M. P. van Exter, M. Travagnin, and J. Woerdman, Opt. Commun. 133, 252 (1997).

${ }^{12}$ D. V. Kuksenkov, H. Temkin, and S. Swirhun, Appl. Phys. Lett. 67, 2141 (1995). 